\title{
The Relationship between Mammography and a Multi-factor Behavioral Index
}

\author{
Andrew R. Marley, MPH \\ Wambui Gathirua-Mwangi, PhD, MPH \\ Michele R. Forman, PhD \\ Timothy E. Stump, MS \\ Patrick Monahan, PhD \\ Victoria L. Champion, RN, PhD, FAAN
}

Objective: In this study, we sought to determine whether a multi-factor behavioral index predicts mammography screening in US women. Methods: Women aged 50-75 years were enrolled in an intervention study and provided their vegetable intake, physical activity (PA), smoking, body mass index (BMI), and alcohol intake. Each factor was scored from 0 (least healthy) to 4 (most healthy) then summed to form a multi-factor behavioral index (ranging from 0-20). Selfreport and medical records were used to determine mammography screening 6-months postintervention. Logistic regression was used to estimate multivariable-adjusted odds ratios (OR) and $95 \%$ confidence intervals $(\mathrm{Cl})$ for the association with 6-month mammography. Results: The mean score for the multi-factor index was 11.8. An increased index score marginally predicted mammography adherence $[O R=1.05(0.99-1.11)]$, with a stronger association among women receiving a doctor's recommendation for mammography [OR $=1.12(1.04-1.20)]$. Of the independent behavioral factors, high PA [OR $=1.13(0.99-1.30), p=.075]$ and low BMI $[O R=1.25$ (1.04-1.51), $p=.017$ ] were marginal and significant predictors of mammography, respectively. Conclusion: Women who engaged in a healthier behavioral lifestyle were more likely to utilize mammography screening, especially if they received a physician recommendation. Physicians should encourage mammography screening as part of a healthy behavioral lifestyle.

Key words: body mass index (BMI); breast health; healthy lifestyle index; mammography; multi-factor behavioral index; women's health

Health Behav Policy Rev. ${ }^{\text {TM }}$ 2019;6(6):582-596

DOI: https://doi.org/10.14485/HBPR.6.6.4

$\mathrm{B}$ reast cancer $(\mathrm{BC})$ is the most common cancer diagnosis and second leading cause of cancer death among women in the United States (US). ${ }^{1}$ In 2019, there will be an estimated 268,600 incident BC cases, and almost 42,000 BC deaths among women in the US, representing $30 \%$ and $15 \%$ of total cancer incidence and deaths, respectively. ${ }^{1}$ Mammography screening has been shown to reduce $\mathrm{BC}$ mortality. ${ }^{2-5}$ Specifically, annual mammography among women aged 50-74 is estimated to reduce $\mathrm{BC}$ mortality by $23 \% .{ }^{6}$ Despite this evi- dence, less than $72 \%$ of US women aged $>50$ years are compliant with mammography screening, a rate far below national goals proposed by Healthy People $2020 .^{7}$ If rates are to increase, it is necessary to investigate screening behaviors and elucidate factors that may influence screening decisions.

Several behavioral factors, including diet, ${ }^{8}$ physical activity (PA), ${ }^{9}$ smoking, ${ }^{10-11}$ alcohol intake, ${ }^{12}$ and body mass index (BMI) ${ }^{13}$ have been repeatedly associated with $\mathrm{BC}$ risk, with some factors possibly having a greater role in the etiology of some

Andrew R. Marley, Indiana University Richard M. Fairbanks School of Public Health, Department of Epidemiology, Indianapolis, IN. Wambui Gathirua-Mwangi, Indiana University School of Nursing, Department of Community Health Systems, Indianapolis, IN. Michele R. Forman, Purdue University, Department of Nutrition, West Lafayette, IN. Timothy E. Stump and Patrick Monahan, Indiana University School of Medicine and Richard M. Fairbanks School of Public Health, Department of Biostatistics, Indianapolis, IN. Victoria L. Champion, Indiana University School of Nursing, Department of Community and Health Systems, Indianapolis, IN.

Correspondence Mr Marley; armarley@iu.edu 
BC subtypes more than others. ${ }^{14-21}$ However, less is known about whether a healthy behavioral lifestyle, demonstrated by these behavioral variables, influences the decision to engage in mammography screening. Specifically, evidence suggests that smoking, alcohol intake, PA, and dietary behaviors may influence mammography uptake; ${ }^{22-24}$ however, the association between individual modifiable behavioral factors and mammography utilization warrants further investigation. Furthermore, a multi-factor behavioral approach may be more informative and elucidating than investigating individual factors. ${ }^{25}$ To our knowledge, a multi-factor behavioral index approach has not been used to predict mammography screening. Such a method has been used to predict mortality, ${ }^{25-28}$ obesity, ${ }^{29}$ all cancer risk ${ }^{25,30,31}$ risk of diabetes, ${ }^{32}$ stroke, ${ }^{33}$ colorectal cancer, ${ }^{43-39}$ gastric cancer ${ }^{40}$ breast cancer, ${ }^{41-43}$ and other outcomes, ${ }^{44-51}$ but not in relation to any cancer screening behavior.

The purpose of this study was to investigate how lifestyle behavioral factors, combined into a single multi-factor behavioral index, relate to mammography screening behavior. Additionally, as receiving a physician recommendation has been a significant factor in previous mammography screening studies, ${ }^{52-54}$ we assessed whether having a physician recommendation modified the relationship between the index and mammography screening. This is the first study to determine if a behavioral lifestyle index is related to cancer screening behavior. We hypothesized that a higher index score, indicating an overall healthier behavioral lifestyle, will be positively associated with mammography uptake, and receipt of a physician recommendation would strengthen this association. Results of this study will inform healthcare providers about lifestyle behavioral factors that influence screening behavior and help promote a healthy lifestyle.

\section{METHODS}

\section{Study Design and Population}

A prospective, randomized factorial colorectal cancer (CRC) screening intervention was conducted in Indiana during 2013-2015, as described in detail elsewhere. ${ }^{55}$ Briefly, women aged $50-75$ years were eligible if they had Internet access and were not adherent to colorectal cancer screening guidelines. Internet access was required for delivery of the intervention. All women recruited were non-adherent to CRC screening, and accrual was stratified by adherence to mammography screening. Therefore, approximately half of the accrued participants were adherent to mammography screening at baseline while the other half were not. If women were non-adherent to both mammography and CRC screening, the intervention addressed both behaviors. Baseline non-adherence to $\mathrm{BC}$ screening was defined as not having a mammogram in the last 15 months. All potentially eligible women had a primary care physician and were identified from 2 community-based healthcare systems' family and internal medicine practices. The list of eligible women was forwarded to Indiana University's Survey Center. Survey center staff accrued participants, obtained consent, and completed data collection. Among 1716 eligible women contacted for the study, 1196 were enrolled after 431 actively refused and 89 refused passively. Participants were then randomized into 4 groups to determine how effective different tailored interventions were at increasing CRC screening relative to usual care. Microsoft SQL random ordering functions were used to perform randomization. Groups were: $\mathrm{N}=305$ in usual care, $\mathrm{N}=303$ in Web-based intervention, $\mathrm{N}=296$ in phone counseling, or $\mathrm{N}=$ 292 in Web-based and phone counseling. Of those enrolled, 275 were lost to follow-up, leaving 921 women for whom outcome data were available. We used a standard questionnaire, administered over the phone by Survey Center staff, to record demographic information, family history, cancer screening history (including mammography), and information regarding alcohol intake, smoking, PA, and vegetable intake. Participants also were given the option to complete this questionnaire online. Self-reported screening behavior also was collected either over the phone or online. At 6 months post-intervention, both self-report and medical record data were used to assess screening behavior for mammography and colorectal screening. This paper focuses only on mammography screening. A participant was considered to have completed screening if either self-report or medical record indicated that screening had occurred. If a participant's self-report conflicted with their medical record, the participant was coded as having screened because medical records may not always be the gold standard, such as if screening occurs outside of the primary healthcare system. 


\section{Multi-factor Behavioral Index}

The focus of this current study was to identify predictors of 6-month mammography screening by using a multi-factor behavioral index. The multifactor behavioral index was created using health behavior data reported by participants at baseline. Specifically, participants' vegetable intake, PA, smoking behavior, alcohol consumption, and BMI were included in the index. Mammography status was measured at 6-months post-intervention. Of the 1196 women initially enrolled, 278 were excluded due to loss to follow-up and missing mammography data. These 278 did not significantly differ regarding age $(\mathrm{p}=.065)$, race $(\mathrm{p}=.12)$, or education level $(\mathrm{p}=.15)$. Of the 918 remaining, 243 were excluded due to missing lifestyle behavior data, leaving 675 with complete health behavior data to construct the index. The 243 excluded did not significantly differ according to age $(\mathrm{p}=$ .51 ), but did significantly differ by education ( $\mathrm{p}$ $=.0018)$ and race $(\mathrm{p}=.0002)$. Specifically, those excluded were less likely to have a college degree and more likely to identify as African-American. Each health behavior variable, except smoking (yes or no), was scored from $0-4$, with higher scores reflecting healthier behavior. Total index scores ranged from $0-20$. As data permitted, the index was scored according to guidelines published by the US Centers for Disease Control and Prevention (CDC) and the US Department of Health and Human Services.

Vegetable intake. Vegetable intake was measured by asking: "In the past 30 days, about how many servings per week of vegetables or leafy green salads did you eat?" and "In the past 30 days, how much did you usually eat in each serving of vegetables or leafy green salads?" According to the $8^{\text {th }}$ edition of Dietary Guidelines for Americans 2015-2020, ${ }^{56}$ adults should consume 2.5 cups of vegetables per day, equating to 17.5 cups of vegetables per week. A score of 4 was given to those who met or exceeded this national recommendation; a score of 3 if their vegetable intake was between 17.5 cups and 13.125 cups (75\% of recommended intake). A score of 2 was given to those with an intake between $50 \%$ and $<75 \%$ of the recommended intake, a score of 1 to those who consumed between $25 \%$ and $<50 \%$ of the recommended intake, and those who had a vegetable intake less than $25 \%$ of the national recommendation received a score of 0 .

Physical activity. Physical activity was measured by asking: "During the last 7 days, how much time did you spend doing vigorous activity?" The CDC recommends that adults perform 150 minutes of vigorous PA per week to achieve the greatest health benefits. ${ }^{57}$ Based on these guidelines, study participants received a score of 4 if they were fully compliant with the 150-minute recommendation, and a score of 3 if their vigorous activity was less than 150 minutes but greater than 112.5 minutes $(75 \%$ of recommendation). Scores of 2, 1 , and 0 were given to those who performed 50 to $<75 \%, 25$ to $<50 \%$, and less than $25 \%$ of the 150 -minute recommendation, respectively.

Alcohol consumption. Alcohol consumption was measured by asking participants: "How often do you currently drink alcoholic beverages?" Despite the elucidation of some potential health benefits, ${ }^{58,59}$ many studies have revealed that alcohol intake increases risk of several serious diseases in a dose-response fashion. ${ }^{60-64}$ This risk been found in association with as little as one drink per day or less ${ }^{64-66}$ leading some to conclude that there is no safe amount of alcohol consumption with respect to mortality from all causes, particularly cancer. $^{62,67}$ Our scoring for alcohol intake reflected this conclusion. Specifically, non-drinkers received a score of 4 and participants who reported drinking alcoholic beverages less than once per week were assigned a score of 3. Participants who consumed alcohol once or twice a week were given a score of 2 , and those who reported alcohol consumption 3-4 times a week received a score of 1 . Participants who reported daily or almost daily consumption of alcohol were assigned a score of 0 .

BMI and smoking. Participants' BMI was measured in units of $\mathrm{kg} / \mathrm{m}^{2}$, and BMI scores followed classifications defined by the CDC. ${ }^{68}$ Participants with a BMI less than 25 received a score of 4; those classified as overweight (BMI 25 to $<30$ ) were assigned a score of 3 ; those with class I obesity (BMI 30 to $<35)$ received a score of 2; participants who were class II obese (BMI 35 to <40) were given a score of 1 ; and participants with class III obesity (BMI of 40 of higher) were assigned a score of 0 . Smoking was measured by asking: "Do you smoke?" Smokers were given a score of 0 , nonsmokers were given a score of 4 . 
Table 1

Characteristics of 50-75-year-old Midwestern Women by 6-month Mammography Screening Status

\begin{tabular}{|c|c|c|c|c|}
\hline Characteristics & Total $(N=675)$ & $\begin{array}{c}\text { Mammography } \\
\text { Screening } \\
(\mathrm{N}=\mathbf{2 7 9 )}\end{array}$ & $\begin{array}{c}\text { No Mammogra- } \\
\text { phy Screening } \\
(\mathrm{N}=396)\end{array}$ & p-value \\
\hline Age [mean (SD); median] ${ }^{\mathrm{a}}$ & $58.8(6.2) ; 57.2$ & $58.7(6.2) ; 56.8$ & $58.9(6.1) ; 57.5$ & .65 \\
\hline Age Range $^{\mathrm{a}}$ & $50.5-75.6$ & $50.5-74.5$ & $50.5-75.6$ & \\
\hline $\begin{array}{l}\text { Multi-factor Behavioral Index Score (mean (SD)) } \\
\text { Range }\end{array}$ & $\begin{array}{c}11.8(3.4) ; 12 \\
3-20\end{array}$ & $\begin{array}{c}12.2(3.1) ; 12 \\
4-19\end{array}$ & $\begin{array}{l}11.5(3.6) ; 11 \\
3-20\end{array}$ & .011 \\
\hline Vegetable intake score (mean; median) & $1.4 ; 1$ & $1.4 ; 1$ & $1.4 ; 1$ & .57 \\
\hline Physical activity score (mean; median) & $1.7 ; 1$ & $1.8 ; 1$ & $1.6 ; 1$ & .092 \\
\hline Smoking score (mean; median) & $3.4 ; 4$ & $3.5 ; 4$ & $3.2 ; 4$ & .010 \\
\hline Alcohol intake score (mean; median) & $2.9 ; 3$ & $2.9 ; 3$ & $2.9 ; 3$ & .93 \\
\hline Body Mass Index score (mean; median) & $2.4 ; 3$ & $2.4 ; 3$ & $2.3 ; 3$ & .38 \\
\hline \multicolumn{5}{|l|}{ Race } \\
\hline White & $602(89.2 \%)$ & $245(87.8 \%)$ & $357(90.2 \%)$ & \\
\hline Black & $58(8.6 \%)$ & $27(9.7 \%)$ & $31(7.8 \%)$ & \\
\hline Other & $15(2.2 \%)$ & $7(2.5 \%)$ & $8(2.0 \%)$ & .63 \\
\hline \multicolumn{5}{|l|}{ Ethnicity } \\
\hline Hispanic/Latino & $5(0.7)$ & $4(1.4 \%)$ & $1(0.3 \%)$ & \\
\hline Non-Hispanic/Latino & $670(99.3 \%)$ & $275(98.6 \%)$ & $395(99.8 \%)$ & .078 \\
\hline \multicolumn{5}{|l|}{ Education } \\
\hline High school graduate or less & $167(24.7 \%)$ & $78(28.0 \%)$ & $89(22.5 \%)$ & \\
\hline Some college/associates/trade school & $275(40.7 \%)$ & $102(36.6 \%)$ & $173(43.7 \%)$ & \\
\hline Bachelor's degree or higher & $233(34.5 \%)$ & $99(35.5 \%)$ & $134(33.8 \%)$ & .13 \\
\hline \multicolumn{5}{|l|}{ Income $^{b}$} \\
\hline$\$ 30,000$ or less & $170(25.8 \%)$ & $64(23.7 \%)$ & $106(27.3 \%)$ & \\
\hline$\$ 30,001-\$ 75,000$ & $286(43.5 \%)$ & $120(44.4 \%)$ & $166(42.8 \%)$ & \\
\hline$\$ 75,000$ or more & $202(30.7 \%)$ & $86(31.9 \%)$ & $116(29.9 \%)$ & .58 \\
\hline \multicolumn{5}{|l|}{ Insurance Coverage $^{c}$} \\
\hline No health insurance & $54(8.1 \%)$ & $16(5.8 \%)$ & $38(9.7 \%)$ & \\
\hline Insurance (Yes) & $614(91.9 \%)$ & $262(94.2 \%)$ & $352(90.3 \%)$ & .062 \\
\hline \multicolumn{5}{|l|}{ Physician Recommendation $^{\mathrm{d}}$} \\
\hline Yes & $343(56.4 \%)$ & $189(72.7 \%)$ & $154(44.3 \%)$ & \\
\hline No & $249(41.0 \%)$ & $68(26.2 \%)$ & $181(52.0 \%)$ & \\
\hline Not sure & $16(2.6 \%)$ & $3(1.2 \%)$ & $\begin{array}{l}13(3.7 \%) \\
\text { (continued on }\end{array}$ & $\begin{array}{l}<.0001 \\
\text { ext page) }\end{array}$ \\
\hline
\end{tabular}

\section{Data Analysis}

Mammography screeners and non-screeners were analyzed for differences in age, race, education, income, insurance coverage, physician recommendation for screening, family history of BC, personal history of $\mathrm{BC}$, previous mammography attendance (ever vs never), and average index score. Differences in categorical and continuous variables were analyzed using chi-square and 2-sided t-tests, respectively. We estimated the association 
Table 1 (continued)

Characteristics of 50-75-year-old Midwestern Women by 6-month Mammography Screening Status

\begin{tabular}{|c|c|c|c|c|}
\hline Characteristics & Total $(N=675)$ & $\begin{array}{c}\text { Mammography } \\
\text { Screening } \\
(\mathrm{N}=\mathbf{2 7 9 )}\end{array}$ & $\begin{array}{c}\text { No Mammogra- } \\
\text { phy Screening } \\
(\mathrm{N}=396)\end{array}$ & p-value \\
\hline \multicolumn{5}{|l|}{ Family History of Breast Cancer } \\
\hline Yes & $140(20.7 \%)$ & $75(26.9 \%)$ & $65(16.4 \%)$ & \\
\hline No & $516(76.4 \%)$ & $194(69.5 \%)$ & $322(81.3 \%)$ & \\
\hline Not sure & $19(2.8 \%)$ & $10(3.6 \%)$ & $9(2.3 \%)$ & .0018 \\
\hline \multicolumn{5}{|c|}{ Personal History of Breast Cancer } \\
\hline Yes & $36(5.3 \%)$ & $24(8.6 \%)$ & $12(3.0 \%)$ & \\
\hline No & $638(94.7 \%)$ & $254(91.4 \%)$ & $384(97.0 \%)$ & .0014 \\
\hline \multicolumn{5}{|l|}{ Previous Mammography } \\
\hline Ever & $643(95.3 \%)$ & $273(97.9 \%)$ & $370(93.4 \%)$ & \\
\hline Never & $32(4.74 \%)$ & $6(2.2 \%)$ & $26(6.6 \%)$ & .0079 \\
\hline \multicolumn{5}{|l|}{ Intervention Group } \\
\hline Web intervention & $161(23.9 \%)$ & $68(24.4 \%)$ & $93(23.5 \%)$ & \\
\hline Phone intervention & $186(27.6 \%)$ & $77(27.6 \%)$ & $109(27.5 \%)$ & \\
\hline Web and phone intervention & $146(21.6 \%)$ & $69(24.7 \%)$ & $77(19.4 \%)$ & \\
\hline Usual care (no interventions) & $182(27.0 \%)$ & $65(23.3 \%)$ & $117(29.6 \%)$ & .21 \\
\hline
\end{tabular}

Note.

Data shown are mean (SD) for continuous variables or $\mathrm{N}(\%)$ for categorical variables; $t$-tests were used to compare continuous variables; chi-square tests were used to compare categorical variables.

a: Age shown in years

b: 17 missing observations - 9 from screeners, 8 from non-screeners

c: 7 missing observations -1 from screeners, 6 from non-screeners

d: 67 missing observations -19 from screeners, 48 from non-screeners

e: One missing observation from screeners

Some percentages do not add up to $100 \%$ due to rounding

between the multi-factor behavioral index and mammography screening. Also, because we previously reported that physician recommendation was a statistically significant factor in cancer screening adherence, ${ }^{54}$ we performed stratified analyses to assess the association between our index and mammography screening according to whether or not participants received a physician recommendation during the study. Finally, we tested each individual behavior in the index, and each of the scores for each behavior, to investigate associations with mammography screening. Smoking was treated as a categorical variable, all others were treated as continuous variables and further explored as categorical variables for dose-response relationships with mammography screening. Logistic regression was used to estimate the odds ratios (OR) and 95\% confidence intervals (CI) and associated p-values. Two models were constructed for the regression analyses. The first model estimated crude associations, the second estimated associations after adjusting for mammography screening covariates: age, education, income, insurance coverage, family history of $\mathrm{BC}$, personal history of $\mathrm{BC}$, previous mammography, physician recommendation of mammography and study intervention in our 
Table 2

Behavioral Factor Scores for Midwestern Women Aged 50-75 Years

\begin{tabular}{cccccc}
\hline & \multicolumn{5}{c}{ Behavioral Factor } \\
\cline { 2 - 6 } Score & Vegetable intake & Physical activity & Smoking & Alcohol intake & BMI \\
\hline 0 & $206(30.5 \%)$ & $313(46.4 \%)$ & $108(16.0 \%)$ & $36(5.3 \%)$ & $93(13.8 \%)$ \\
1 & $236(35.0 \%)$ & $65(9.6 \%)$ & -- & $60(8.9 \%)$ & $90(13.3 \%)$ \\
2 & $112(16.6 \%)$ & $20(3.0 \%)$ & -- & $91(13.5 \%)$ & $133(19.7 \%)$ \\
3 & $10(1.5 \%)$ & $70(10.4 \%)$ & -- & $207(30.7 \%)$ & $186(27.6 \%)$ \\
4 & $111(16.4 \%)$ & $207(30.7 \%)$ & $567(84.0 \%)$ & $281(41.6 \%)$ & $173(25.6 \%)$ \\
Mean; Median & $1.38 ; 1.00$ & $1.69 ; 1.0$ & $3.36 ; 4.00$ & $2.94 ; 3.00$ & $2.38 ; 3.00$ \\
\hline
\end{tabular}

Note.

Score: $0=$ Unhealthy behavior (Not meeting recommended guidelines of the lifestyle factor), and $4=$ Healthy behavior (Meeting the recommended guidelines of the lifestyle factor).

Data shown are $\mathrm{N}(\%)$ of participants receiving each score for the differing lifestyle factors.

original study. ${ }^{55}$ Stratified analyses did not include physician recommendation in the adjusted model. We controlled for the intervention to minimize any possible confounding of our results by intervention content. When analyzing the individual health behaviors within the index, a third model was constructed to additionally adjust for the other health behavior variables. To assess whether physician rec- ommendation for screening modified the association between the multi-factor behavioral index and mammography, an interaction term of the index score and physician recommendation was included in the crude and adjusted models. All analyses were performed using SAS, version 9.4 (SAS Institute Inc., Cary, NC). All p-values <.05 (2-sided) were considered statistically significant.

Table 3

Association between Multi-factor Behavioral Index and 6-month Mammography Screening among Midwestern Women aged 50-75, and Stratified by Physician Recommendation

\begin{tabular}{|c|c|c|c|}
\hline Lifestyle Index & Odds Ratio & $95 \% \mathrm{CI}$ & p-value \\
\hline \multicolumn{4}{|l|}{ All Participants } \\
\hline Crude $(\mathrm{N}=675)$ & 1.06 & $1.01-1.11$ & .013 \\
\hline Adjusted $^{\mathrm{a}}(\mathrm{N}=586)$ & 1.05 & $0.99-1.11$ & .083 \\
\hline \multicolumn{4}{|c|}{ No Physician Recommendation } \\
\hline Crude $(\mathrm{N}=249)$ & 0.96 & $0.89-1.04$ & .35 \\
\hline Adjusted $^{\mathrm{b}}(\mathrm{N}=236)$ & 0.95 & $0.86-1.04$ & .25 \\
\hline \multicolumn{4}{|c|}{ Physician Recommendation } \\
\hline Crude $(\mathrm{N}=343)$ & 1.12 & $1.05-1.20$ & .0009 \\
\hline Adjusted $^{\mathrm{b}}(\mathrm{N}=334)$ & 1.12 & $1.04-1.20$ & .0027 \\
\hline
\end{tabular}

Note.

a: Adjusted for age, education, income, health insurance coverage, family history of breast cancer, personal history of breast cancer, previous mammography, intervention group, and physician recommendation.

b: Adjusted for age, education, income, health insurance coverage, family history of breast cancer, personal history of breast cancer, previous mammography, and intervention group. 


\section{Table 4}

\section{Association between Individual Lifestyle Behaviors and 6-month Mammography} Screening among Midwestern Women Aged 50-75

\begin{tabular}{|c|c|c|c|c|c|c|}
\hline & \multicolumn{6}{|c|}{ Model } \\
\hline & Unstratified & $\begin{array}{c}\text { p- } \\
\text { value }\end{array}$ & $\begin{array}{c}\text { Stratified } \\
\text { no physician } \\
\text { recommendation }\end{array}$ & $\begin{array}{c}\text { p- } \\
\text { value }\end{array}$ & $\begin{array}{c}\text { Stratified } \\
\text { physician } \\
\text { recommendation }\end{array}$ & $\begin{array}{c}\text { p- } \\
\text { value }\end{array}$ \\
\hline \multicolumn{7}{|l|}{ Vegetable Intake } \\
\hline Crude OR $(95 \% \mathrm{CI})$ & $1.03(0.92-1.16)$ & .57 & $0.91(0.73-1.12)$ & .39 & $1.06(0.91-1.24)$ & .44 \\
\hline Adjusted OR1 $(95 \% \mathrm{CI})^{\mathrm{a}}$ & $0.96(0.84-1.10)$ & .54 & $0.86(0.67-1.09)$ & .23 & $1.01(0.86-1.20$ & .88 \\
\hline Adjusted OR2 $(95 \% \mathrm{CI})^{\mathrm{b}}$ & $0.94(0.82-1.08)$ & .36 & $0.86(0.67-1.09)$ & .22 & $0.98(0.82-1.16)$ & .80 \\
\hline \multicolumn{7}{|l|}{ Physical activity } \\
\hline Crude OR $(95 \% \mathrm{CI})$ & $1.08(0.99-1.17)$ & .09 & $0.95(0.81-1.11)$ & .54 & $1.18(1.05-1.34)$ & .007 \\
\hline Adjusted OR1 $(95 \% \mathrm{CI})^{\mathrm{a}}$ & $1.10(0.99-1.21)$ & .06 & $0.96(0.81-1.14)$ & .67 & $1.19(1.04-1.35)$ & .010 \\
\hline Adjusted OR2 $(95 \% \mathrm{CI})^{\mathrm{b}}$ & $1.08(0.97-1.20)$ & .16 & $0.99(0.82-1.18)$ & .87 & $1.13(0.99-1.30)$ & .075 \\
\hline \multicolumn{7}{|l|}{ Smoking } \\
\hline Crude OR $(95 \% \mathrm{CI})$ & $1.15(1.03-1.29)$ & .01 & $1.16(0.56-2.54)$ & .70 & $1.17(1.01-1.38)$ & .046 \\
\hline Adjusted OR1 $(95 \% \mathrm{CI})^{\mathrm{a}}$ & $1.10(0.96-1.25)$ & .17 & $1.02(0.84-1.27)$ & .82 & $1.16(0.98-1.38)$ & .088 \\
\hline Adjusted OR2 $(95 \% \mathrm{CI})^{\mathrm{b}}$ & $1.10(0.97-1.26)$ & .15 & $1.02(0.83-1.28)$ & .85 & $1.19(1.00-1.43)$ & .057 \\
\hline \multicolumn{7}{|l|}{ Alcohol Consumption } \\
\hline Crude OR $(95 \% \mathrm{CI})$ & $0.99(0.87-1.13)$ & .93 & $0.95(0.76-1.20)$ & .69 & $0.88(0.72-1.06)$ & .17 \\
\hline Adjusted OR1 $(95 \% \mathrm{CI})^{\mathrm{a}}$ & $0.96(0.82-1.12)$ & .58 & $0.97(0.75-1.26)$ & .80 & $0.92(0.74-1.14)$ & .46 \\
\hline Adjusted OR2 $(95 \% \mathrm{CI})^{\mathrm{b}}$ & $0.98(0.83-1.16)$ & .84 & $0.91(0.69-1.20)$ & .50 & $0.96(0.77-1.20)$ & .73 \\
\hline \multicolumn{7}{|l|}{ BMI } \\
\hline Crude OR $(95 \% \mathrm{CI})$ & $1.06(0.94-1.18)$ & .38 & $0.93(0.75-1.14)$ & .46 & $1.30(1.11-1.53)$ & .002 \\
\hline Adjusted OR1 $(95 \% \mathrm{CI})^{\mathrm{a}}$ & $1.11(0.97-1.27)$ & .13 & $0.88(0.70-1.11)$ & .29 & $1.28(1.07-1.52)$ & .006 \\
\hline Adjusted OR2 $(95 \% \mathrm{CI})^{\mathrm{b}}$ & $1.09(0.95-1.26)$ & .23 & $0.86(0.67-1.11)$ & .24 & $1.25(1.04-1.51)$ & .017 \\
\hline
\end{tabular}

Note.

OR, odds ratio; CI, confidence interval

a: Adjusted for age, education, income, health insurance coverage, family history of breast cancer, personal history of breast cancer, previous mammography, physician recommendation (unstratified only), and intervention b: Additionally adjusted for other health behaviors in index

c: Treated as categorical in the model because it contains only 2 values ( 0 or 4$)$; all other variables (vegetable, physical activity, alcohol consumption and BMI) were treated as continuous in Table 4 and further explored for categorical dose-response relationships in Table 5

\section{RESULTS}

Participants were predominantly white $(89.2 \%)$, with a mean (median) age of 58.8 (57.2) years, and an average index score of 11.76 (range of 3-20). Overall, $41.3 \%$ of the women in our sample were adherent to mammography guidelines. Women who were adherent to mammography by 6 -months post-intervention were more likely to have a higher multi-factor behavioral index, health insurance, a family history of $\mathrm{BC}$, a personal history of $\mathrm{BC}$, and have had a previous mammogram compared to those who did not screen. Among women who screened, $72.7 \%$ received a physician's recommendation relative to $44.3 \%$ among those who did not undergo screening (Table 1 ). The majority of women consumed either less than $25 \%(30.5 \%)$ or 25 to $<50 \%(35.0 \%)$ of the recommended vegetable intake per week (Table 2). Additionally, 46.4\% of women performed $25 \%$ or less of the national vigorous activity recommended per week. Most 
Table 5

Association between Scores for Individual Behavioral Factors and 6-month Mammography Screening among Midwestern Women aged 50-75 Years by Physician Recommendation to Screen Status

\begin{tabular}{|c|c|c|c|c|c|}
\hline \multirow[b]{2}{*}{ Nutrients } & \multicolumn{4}{|c|}{ Index Score ${ }^{\mathrm{c}}$} & \multirow[b]{2}{*}{ Four } \\
\hline & $\begin{array}{l}\text { Zero } \\
\text { (ref) }\end{array}$ & One & Two & Three & \\
\hline \multicolumn{6}{|c|}{ Participants who received a Physician recommendation to screen } \\
\hline \multicolumn{6}{|l|}{ Vegetable intake } \\
\hline Crude OR $(95 \% \mathrm{CI})$ & 1.00 & $1.36(0.80-2.32)$ & $1.88(0.98-3.66)$ & $1.04(0.19-5.83)$ & $1.23(0.65-2.34)$ \\
\hline Adjusted OR1 $(95 \% \mathrm{CI})^{\mathrm{a}}$ & 1.00 & $1.17(0.66-2.08)$ & $1.42(0.70-2.94)$ & $0.85(0.15-5.00)$ & $1.05(0.53-2.10)$ \\
\hline Adjusted OR2 $(95 \% \mathrm{CI})^{\mathrm{b}}$ & 1.00 & $1.19(0.65-2.18)$ & $1.28(0.61-2.73)$ & $0.47(0.08-2.93)$ & $0.96(0.47-1.98)$ \\
\hline \multicolumn{6}{|l|}{ Physical activity } \\
\hline Crude OR $(95 \% \mathrm{CI})$ & 1.00 & $0.89(0.41-1.88)$ & $1.86(0.54-7.33)$ & 3.32 (1.47-8.27) & $1.68(1.02-2.78)$ \\
\hline Adjusted OR1 $(95 \% \text { CI })^{\mathrm{a}}$ & 1.00 & $0.79(0.35-1.76)$ & $1.45(0.38-6.04)$ & 3.34 (1.42-8.62) & $1.82(0.92-3.67)$ \\
\hline Adjusted OR2 $(95 \% \mathrm{CI})^{\mathrm{b}}$ & 1.00 & $0.71(0.31-1.61)$ & $1.29(0.33-5.53)$ & 2.73 (1.07-7.49) & $1.41(0.80-2.52)$ \\
\hline \multicolumn{6}{|l|}{ Smoking } \\
\hline Crude OR $(95 \%$ CI) & 1.00 & d & d & d & $1.90(1.02-3.62)$ \\
\hline Adjusted OR1 $(95 \% \text { CI })^{\mathrm{a}}$ & 1.00 & d & d & d & $1.79(0.91-3.61)$ \\
\hline Adjusted OR2 $(95 \% \mathrm{CI})^{\mathrm{b}}$ & 1.00 & d & d & d & $1.80(0.87-3.82)$ \\
\hline \multicolumn{6}{|l|}{ Alcohol consumption } \\
\hline Crude OR $(95 \%$ CI) & 1.00 & $1.48(0.42-5.33)$ & $1.72(0.55-5.38)$ & $1.11(0.39-3.14)$ & $0.91(0.33-2.50)$ \\
\hline Adjusted OR1 $(95 \% \mathrm{CI})^{\mathrm{a}}$ & 1.00 & $1.15(0.30-4.47)$ & $1.53(0.46-5.07)$ & $1.04(0.34-3.16)$ & $0.93(0.31-2.78)$ \\
\hline Adjusted OR2 $(95 \% \mathrm{CI})^{\mathrm{b}}$ & 1.00 & $0.91(0.22-3.79)$ & $1.41(0.39-5.13)$ & $1.01(0.30-3.34)$ & $0.89(0.27-2.89)$ \\
\hline \multicolumn{6}{|l|}{ BMI } \\
\hline Crude OR (95\% CI) & 1.00 & $1.30(0.59-2.90)$ & $2.29(1.11-4.84)$ & $2.11(1.04-4.33)$ & $3.04(1.46-6.49)$ \\
\hline Adjusted OR1 $(95 \% \text { CI })^{\mathrm{a}}$ & 1.00 & $1.43(0.61-3.41)$ & $2.66(1.20-6.02)$ & $2.13(1.00-4.65)$ & 3.02 (1.36-6.87) \\
\hline Adjusted OR2 $(95 \% \mathrm{CI})^{\mathrm{b}}$ & 1.00 & $1.17(0.48-2.87)$ & $2.41(1.05-5.63)$ & $1.94(0.87-4.44)$ & $2.95(1.24-7.17)$ \\
\hline
\end{tabular}

women were non-smokers $(84.0 \%)$, and the most prevalent categories for alcohol intake and BMI were abstinence $(41.6 \%)$ and 25 to $<30(27.6 \%)$, respectively.

Our multi-factor behavioral index was significantly associated with mammography use in the crude model and marginally associated in the adjusted model (Table 3). After adjustment for covariates, study participants with a higher index score were marginally more likely to receive mammography screening [OR=1.05 (0.99-1.11), $\mathrm{p}=.084]$ (Table $3)$. Behavioral index scores did not significantly differ among those who did and did not receive a physician recommendation to screen $(p=.63)$; however, physician recommendation significantly modified the association between the multi-factor behavioral index and mammography association (interaction $\mathrm{p}$-value $=.002$ ). Therefore, separate logistic regression models were run for recommendation and no recommendation from physician (Table 3). After adjusting for covariates, women with a higher index score, who also received a doctor's recommendation for mammography, were more likely to screen $[\mathrm{OR}=1.12(1.04-1.20), \mathrm{p}$ $=.0027]$. No behavior-mammography association was observed among women who did not receive a physician's recommendation in crude or adjusted models [adjusted OR $=0.95$ (0.86-1.04), $\mathrm{p}=.25$ ]

Of the individual behavioral factors within the index, vegetable intake $(\mathrm{p}=.80)$ and alcohol con- 
Table 5 (continued)

Association between Scores for Individual Behavioral Factors and 6-month Mammography Screening among Midwestern Women aged 50-75 Years by Physician Recommendation to Screen Status

\begin{tabular}{|c|c|c|c|c|c|}
\hline \multirow[b]{2}{*}{ Nutrients } & \multicolumn{4}{|c|}{ Index Score $^{\mathrm{c}}$} & \multirow[b]{2}{*}{ Four } \\
\hline & $\begin{array}{c}\text { Zero } \\
\text { (ref) }\end{array}$ & One & Two & Three & \\
\hline \multicolumn{6}{|c|}{ Participants without a Physician recommendation to screen } \\
\hline \multicolumn{6}{|l|}{ Vegetable intake } \\
\hline Crude OR $(95 \% \mathrm{CI})$ & 1.00 & $0.82(0.42-1.63)$ & $0.94(0.40-2.16)$ & e & $0.84(0.35-1.94)$ \\
\hline Adjusted OR1 $(95 \% \mathrm{CI})^{\mathrm{a}}$ & 1.00 & $0.83(0.40-1.74)$ & $0.82(0.30-2.19)$ & e & $0.57(0.20-1.49)$ \\
\hline Adjusted OR2 $(95 \% \mathrm{CI})^{\mathrm{b}}$ & 1.00 & $0.89(0.41-1.92)$ & $0.96(0.32-2.77)$ & e & $0.61(0.20-1.74)$ \\
\hline \multicolumn{6}{|l|}{ Physical activity } \\
\hline Crude OR $(95 \% \mathrm{CI})$ & 1.00 & $1.45(0.51-3.87)$ & $2.02(0.38-9.63)$ & $1.42(0.56-3.46)$ & $0.75(0.38-1.46)$ \\
\hline Adjusted OR1 $(95 \% \mathrm{CI})^{\mathrm{a}}$ & 1.00 & $1.61(0.50-4.93)$ & $2.90(0.45-16.94)$ & $1.59(0.55-4.37)$ & $0.80(0.37-1.66)$ \\
\hline Adjusted OR2 $(95 \% \mathrm{CI})^{\mathrm{b}}$ & 1.00 & $1.51(0.45-4.83)$ & $2.48(0.38-15.00)$ & $1.56(0.52-4.48)$ & $0.79(0.35-1.74)$ \\
\hline \multicolumn{6}{|l|}{ Smoking } \\
\hline Crude OR $(95 \% \mathrm{CI})$ & 1.00 & d & d & d & $1.16(0.56-2.54)$ \\
\hline Adjusted OR1 $(95 \% \mathrm{CI})^{\mathrm{a}}$ & 1.00 & d & d & d & $1.01(0.49-2.63)$ \\
\hline Adjusted OR2 $(95 \% \mathrm{CI})^{\mathrm{b}}$ & 1.00 & d & d & d & $1.14(0.47-2.93)$ \\
\hline \multicolumn{6}{|l|}{ Alcohol consumption } \\
\hline Crude OR $(95 \%$ CI) & 1.00 & $2.29(0.55-11.92)$ & $1.44(0.36-7.33)$ & $2.17(0.63-10.03)$ & $1.24(0.36-5.78)$ \\
\hline Adjusted OR1 $(95 \% \mathrm{CI})^{\mathrm{a}}$ & 1.00 & $1.55(0.32-9.18)$ & $1.16(0.25-6.62)$ & $2.18(0.55-11.17)$ & $1.01(0.26-5.15)$ \\
\hline Adjusted OR2 $(95 \% \mathrm{CI})^{\mathrm{b}}$ & 1.00 & $1.71(0.33-10.60)$ & $1.27(0.27-7.38)$ & $1.93(0.47-10.23)$ & $0.83(0.20-4.38)$ \\
\hline \multicolumn{6}{|l|}{ BMI } \\
\hline Crude OR $(95 \% \mathrm{CI})$ & 1.00 & $1.19(0.40-3.69)$ & $1.27(0.46-3.68)$ & $0.88(0.34-2.41)$ & $0.86(0.33-2.36)$ \\
\hline Adjusted OR1 $(95 \% \mathrm{CI})^{\mathrm{a}}$ & 1.00 & $0.98(0.29-3.32)$ & $1.23(0.41-3.81)$ & $0.84(0.30-2.48)$ & $0.64(0.22-1.91)$ \\
\hline Adjusted OR2 $(95 \% \mathrm{CI})^{\mathrm{b}}$ & 1.00 & $0.78(0.21-2.86)$ & $0.96(0.30-3.14)$ & $0.84(0.28-2.63)$ & $0.54(0.16-1.84)$ \\
\hline
\end{tabular}

\section{Note.}

Ref, reference; OR, odds ratio; CI, confidence interval

a: Adjusted for age, education, income, health insurance coverage, family history of breast cancer, personal history of breast cancer, previous mammogram, and intervention

b: Additionally adjusted for other health behavior index scores

c: Index Score: 0 = Unhealthy behavior (Not meeting recommended guidelines of the lifestyle factor), and $4=$ Healthy behavior (Meeting the recommended guidelines of the lifestyle factor)

d: No data - Smoking was scored as 0 (smokers) or 4 (non-smokers)

e: Inadequate observations to generate OR $(95 \% \mathrm{CI})$

sumption $(\mathrm{p}=.73)$ were not associated with mammography in crude or either of the adjusted models, even among those with a physician's recommendation (Table 4). Among those with physician recommendation, smoking was statistically significant in the crude model but only marginally associated with mammography in the model adjusted for co- variates and other lifestyle factors $(\mathrm{p}=.057)$. BMI was a statistically significant predictor of mammography among women who received a physician's recommendation to screen. After adjustment for covariates, including other behavioral factors, women with a low BMI [OR = 1.25 (1.04-1.51), p $=.017$ ] were more likely to screen (Table 4). 
In further exploration of the dose-response relationship for specific scores of each behavioral variable (Table 5), women who received a physician recommendation and had a BMI of: $<25 \mathrm{~kg} / \mathrm{m}^{2}$ $[\mathrm{OR}=2.95(1.24-7.17)]$ and $30-35 \mathrm{~kg} / \mathrm{m}^{2}$ [OR $=2.41(1.05-5.63)$ were more likely to receive a mammogram compared to women with a BMI $>40 \mathrm{~kg} / \mathrm{m}^{2}$. Among women who received a physician's recommendation to screen (Table 4), PA was a statistically significant predictor of mammography in the first adjusted model [OR $=1.19$ (1.04$1.35), \mathrm{p}=.0098]$, and a marginal predictor in the second adjusted model [OR $=1.13(0.99-1.30)$, $\mathrm{p}$ $=.075]$. Specifically, women who received a physician recommendation and practiced PA for 112.5150 minutes/week were more likely to receive a mammogram $[\mathrm{OR}=2.73(1.07-7.49)]$ compared with women who were physically inactive (Table 5).

\section{DISCUSSION}

We report that a multi-factor behavioral index, comprised of vegetable intake, PA, smoking, alcohol intake, and BMI, was marginally associated with mammography screening. However, when combined with a physician recommendation, our index was significantly, positively associated with mammography. Within the index, BMI and PA were significantly and marginally associated with mammography among individuals who received a physician recommendation to screen, respectively, in the overall sample. However, physician recommendation was a strong effect modifier. Among women who received a physician recommendation, those who were more physically active and those with a lower BMI were more likely to screen compared to those who were inactive and had a higher BMI, respectively, after adjusting for baseline covariates and other individual behavioral factors.

To our knowledge, we are the first to utilize a multi-factor behavioral index approach in relation to mammography screening behavior. Such a method has been used to predict cancer risk ${ }^{25,30,31,34-}$ 43 as well as associations with a variety of other health-related outcomes ${ }^{26-29,32,33,44-51}$ but not for cancer screening. Although this method has not been used to predict mammography screening, vegetable intake, ${ }^{22} \mathrm{PA},{ }^{22,69}$ smoking behavior, ${ }^{22,69-80}$ alcohol consumption, ${ }^{22,69,72,79}$ and $\mathrm{BMI}^{22,71-76,81-89}$ have been investigated individually for associations with mammography. Our findings for BMI and PA only among women who received a recommendation for mammography underscores the key role of health providers in increasing screening rates. Our previous studies ${ }^{52-54}$ and those of others ${ }^{90-94}$ show a provider recommendation significantly improves $\mathrm{BC}$ screening rates.

Within our index, BMI was inversely associated with mammography screening. The association between BMI and mammography has been studied extensively in the literature, but results remain inconsistent. In accord with our finding, other studies have suggested an inverse association between BMI and mammography. ${ }^{74,85,86}$ A large, US-based study found that obese women were significantly less likely to screen than normal-weight women an association that was even stronger among women classified as morbidly obese, but no statistically significant results appeared among the overweight women. ${ }^{89}$ In a 2009 meta-analysis, morbidly obese women (BMI > 40), but no other classes of obesity or overweight, were significantly less likely to undergo mammography relative to normal-weight women. ${ }^{95}$ In contrast, other studies produced null results. $22,71-73,75,83$ The possible explanation for reduced screening among obese women may involve embarrassment, perceived weight stigma, lack of appropriately sized examination equipment, or poor patient-provider communication. ${ }^{96}$

We report PA was marginally, positively associated with mammography screening. PA has been found to be associated with mammography in several, ${ }^{22,70,82}$ but not all studies. ${ }^{69,71}$ Furthermore, we found a slightly inverted u-shaped association between PA and mammography (also seen for alcohol consumption although no significant results). A possible explanation for this could be that women with the healthiest PA (and alcohol consumption) behavior considered their behavior as protective against $\mathrm{BC}$, and therefore, did not feel as much of a need to participate in mammography. Our study did not find an association between mammography and the other behavioral factors (vegetable intake, smoking, and alcohol consumption). Relative to BMI and PA, fewer studies have explored the role of vegetable intake and alcohol consumption with respect to $\mathrm{BC}$ screening. Contrary to our findings, Sözmen et al, found those with a high 
vegetable intake were more likely to screen compared to those who did not. ${ }^{70}$ Regarding alcohol intake and BC screening, some studies found no association, ${ }^{69,72}$ whereas others found that alcohol intake was associated with increased ${ }^{22}$ or reduced ${ }^{79}$ screening when compared to those with low or no alcohol intake. Unlike our study and others ${ }^{71,75,80}$ some previous research has found that smokers were less likely to screen for BC compared to nonsmokers. $22,69,72-74,76,77,79$ One difference in our study that may help explain the null association between smoking and mammography is the inability to identify former smokers.

Our results should be interpreted within the context of the study's limitations. In this study, non-adherence to mammography guidelines was defined as not having a mammogram within the previous 15 months. This is because, at the time data for this study were collected, the American Cancer Society's mammography recommendation stated that women should begin annual screening at age 40 and continue with annual mammography for as long as the woman is in reasonably good health. ${ }^{97}$ Data were collected in accordance with screening recommendations for that time. Since this time, the American Cancer Society recommends annual and biennial mammograms for women aged 45-54 years and $\geq 55$ years, respectively. ${ }^{97}$ Although guidelines have changed, our study investigates factors pertaining to mammography adoption, not timing, so we believe our results still to be valid, meaningful, and useful. Demographically, our sample is not representative of the US population of women aged 50-75, detracting from the generalizability of our results. Additionally, those that were excluded due to missing health behavior data statistically differed to those that were included with respect to education and race. Although we adjusted for these variables in all analyses, it is possible that our results may have been different if these women had been included. No biomarkers were used to assess participants' behavioral/lifestyle factors objectively; rather, data were ascertained via participant recall. In some instances, participant recall also was used for assessment of screening behavior, introducing the possibility of misclassification. Additionally, PA was based on participant recall of activity for the previous 7 days. Although these gathered data may have been an accurate representation of typical PA, it is possible that some participants' PA during the time of interview, 2013-2015, was anomalous to their typical or average behavior. Regarding smoking, our data were limited to current smokers and non-smokers only; no data were collected regarding former smoking activity. Regarding alcohol consumption, it is possible that non-drinkers were abstaining from alcohol because of comorbidities or other conditions dictating their drinking behavior rather than due to a desire to be healthy. Limitations to alcohol consumption data prevented us from scoring this behavior using average drinks per day, a preferable measure as CDC recommendations are reported drinks per day. ${ }^{98}$ Also, we were limited by our inability to differentiate diagnostic screening from preventive mammography. Because of this, all screening was classified as mammography, thereby raising the possibility that diagnostic screens were counted as mammography screening.

\section{IMPLICATIONS FOR HEALTH BEHAVIOR OR POLICY}

Our study findings have implications for primary care physicians and other healthcare providers, emphasizing their role in improving national screening rates. Women in our study were more likely to receive mammography screening if they had a healthier behavioral lifestyle and received a physician's recommendation to screen. These results suggest that encouraging a healthier lifestyle and increasing patient-physician communication regarding $\mathrm{BC}$ screening may help to improve mammography adherence among US women. Therefore, to increase nationwide screening rates from less than $72 \%$ to the $81.1 \%$ goal proposed in Healthy People 2020, ${ }^{7}$ healthcare providers should promote mammography screening as part of overall healthy living, especially among women who do not have a healthy behavioral lifestyle.

\section{Acknowledgements}

Research reported in this publication was supported by the National Institutes of Health (R01CA136940-5 to Dr. Victoria Champion). The efforts of Andrew R. Marley and Wambui Gathirua-Mwangi were supported by the National Cancer Institute: (T32CA117865; PI: Victoria L. Champion) and (3R01CA196243-02S1; PIs: Victoria L. Champion and Electra Paskett), respectively. The content is solely the responsibility of 
the authors and does not necessarily represent the official views of the National Institutes of Health. A previous abstract and poster of this research was displayed at the Indiana University Simon Cancer Center Cancer Research Day, a conference in Indianapolis, IN. The authors also thank the Purdue Cancer Center for support of this research.

\section{Human Subjects Approval Statement}

The Institutional Review Board at Indiana University and community sites approved the study, and it is registered with the clinical trials identifier NCT03279198. This study is a secondary analysis of previously-collected data and investigators did not contact or identify study participants.

\section{Conflict of Interest Disclosure Statement}

The authors have no conflicts of interest to declare.

\section{References}

1. American Cancer Society (ACS). Cancer Facts \& Figures 2019. Atlanta, GA: ACS; 2019.

2. Arleo EK, Hendrick RE, Helvie MA, Sickles EA. Comparison of recommendations for screening mammography using CISNET models. Cancer. 2017;123(19):36733680.

3. Tabar L, Gad A, Holmberg L, et al. Reduction in mortality from breast cancer after mass screening with mammography: randomised trial from the Breast Cancer Screening Working Group of the Swedish National Board of Health and Welfare. Lancet. 1985;325(8433):829-832.

4. Otto SJ, Fracheboud J, Looman CW, et al. Initiation of population-based mammography screening in Dutch municipalities and effect on breast-cancer mortality: a systematic review. Lancet. 2003;361(9367):1411-1417.

5. Kalager M, Zelen M, Langmark F, Adami H-O. Effect of screening mammography on breast-cancer mortality in Norway. N Engl J Med. 2010;363(13):1203-1210.

6. Hendrick RE, Helvie MA. United States preventive services task force screening mammography recommendations: science ignored. Am J Roentgenol. 2011;196(2):W112W116.

7. Office of Disease Prevention and Health Promotion. C-17: Women receiving a mammogram within past 2 years (age-adjusted, percent, 50-74). Available at: https:// www.healthypeople.gov/2020/data-search/Search-theData\#objid=4055. Accessed October 17, 2019.

8. Gandini S, Merzenich H, Robertson C, Boyle P. Metaanalysis of studies on breast cancer risk and diet: the role of fruit and vegetable consumption and the intake of associated micronutrients. Eur J Cancer. 2000;36(5):636646.

9. Thune I, Brenn T, Lund E, Gaard M. Physical activity and the risk of breast cancer. $N$ Engl J Med.
1997;336(18):1269-1275.

10. Gaudet MM, Gapstur SM, Sun J, et al. Active smoking and breast cancer risk: original cohort data and metaanalysis. J Natl Cancer Inst. 2013;105(8):515-525.

11. Johnson KC, Miller AB, Collishaw NE, et al. Active smoking and secondhand smoke increase breast cancer risk: the report of the Canadian Expert Panel on Tobacco Smoke and Breast Cancer Risk (2009). Tob Control. 2010;20(1):e2.

12. Smith-Warner SA, Spiegelman D, Yaun S-S, et al. Alcohol and breast cancer in women: a pooled analysis of cohort studies. JAMA. 1998;279(7):535-540.

13. Van Den Brandt PA, Spiegelman D, Yaun S-S, et al. Pooled analysis of prospective cohort studies on height, weight, and breast cancer risk. Am J Epidemiol. 2000;152(6):514527.

14. Zhang L, Huang Y, Feng Z, et al. Comparison of breast cancer risk factors among molecular subtypes: a case-only study. Cancer Med. 2019;8(4):1882-1892.

15. Yang XR, Sherman ME, Rimm DL, et al. Differences in risk factors for breast cancer molecular subtypes in a population-based study. Cancer Epidemiol Biomarkers Prev. 2007;16(3):439-443.

16. Brouckaert O, Van Asten K, Laenen A, et al. Body mass index, age at breast cancer diagnosis, and breast cancer subtype: a cross-sectional study. Breast Cancer Res Treat. 2018;168(1):189-196.

17. Phipps AI, Buist DS, Malone KE, et al. Breast density, body mass index, and risk of tumor marker-defined subtypes of breast cancer. Ann Epidemiol. 2012;22(5):340348.

18. Turkoz FP, Solak M, Petekkaya I, et al. Association between common risk factors and molecular subtypes in breast cancer patients. Breast. 2013;22(3):344-350.

19. Li CI, Chlebowski RT, Freiberg M, et al. Alcohol consumption and risk of postmenopausal breast cancer by subtype: the women's health initiative observational study. J Natl Cancer Inst. 2010;102(18):1422-1431.

20. Schmidt ME, Steindorf K, Mutschelknauss E, et al. Physical activity and postmenopausal breast cancer: effect modification by breast cancer subtypes and effective periods in life. Cancer Epidemiol Biomarkers Prev. 2008;17(12):3402-3410.

21. Kabat GC, Kim M, Phipps AI, et al. Smoking and alcohol consumption in relation to risk of triple-negative breast cancer in a cohort of postmenopausal women. Cancer Causes Control. 2011;22(5):775-783.

22. Lagerlund M, Drake I, Wirfält E, et al. Health-related lifestyle factors and mammography screening attendance in a Swedish cohort study. Eur J Cancer Prev. 2015;24(1):4450.

23. Galán I, Rodríguez-Artalejo F, Díez-Gañán L, et al. Clustering of behavioural risk factors and compliance with clinical preventive recommendations in Spain. Prev Med. 2006;42(5):343-347.

24. Abdullah A, Leung K, Leung C, et al. Factors associated with the use of breast and cervical cancer screening services among Chinese women in Hong Kong. Public Health. 2001;115(3):212-217.

25. Meng L, Maskarinec G, Lee J, Kolonel LN. Lifestyle factors and chronic diseases: application of a composite risk index. Prev Med. 1999;29(4):296-304. 
26. Yun JE, Won S, Kimm H, Jee SH. Effects of a combined lifestyle score on 10-year mortality in Korean men and women: a prospective cohort study. BMC Public Health. 2012;12(1):673.

27. Malekshah AF-t, Zaroudi M, Etemadi A, et al. The combined effects of healthy lifestyle behaviors on all-cause mortality: the Golestan cohort study. Arch Iran Med. 2016;19(11):752.

28. Odegaard AO, Koh W-P, Gross MD, et al. Combined lifestyle factors and cardiovascular disease mortality in Chinese men and women: the Singapore Chinese health study. Circulation. 2011;124(25):2847-2854.

29. Kovács É, Hunsberger M, Reisch L, et al. Adherence to combined lifestyle factors and their contribution to obesity in the IDEFICS study. Obes Rev. 2015;16:138-150.

30. Sasazuki S, Inoue M, Iwasaki M, et al. Combined impact of five lifestyle factors and subsequent risk of cancer: the Japan Public Health Center study. Prev Med. 2012;54(2):112-116.

31. McKenzie F, Biessy C, Ferrari P, et al. Healthy lifestyle and risk of cancer in the European prospective investigation into cancer and nutrition cohort study. Medicine. 2016;95(16).?????Page numbers?????

32. Mozaffarian D, Kamineni A, Carnethon M, et al. Lifestyle risk factors and new-onset diabetes mellitus in older adults: the cardiovascular health study. Arch Intern Med. 2009;169(8):798-807.

33. Rist PM, Buring JE, Kase CS, Kurth T. Healthy lifestyle and functional outcomes from stroke in women. $\mathrm{Am} \mathrm{J}$ Med. 2016;129(7):715-724. e712.

34. Odegaard AO, Koh W-P, Yuan J-M. Combined lifestyle factors and risk of incident colorectal cancer in a Chinese population. Cancer Prev Res (Phila). 2013;6(4):360-367.

35. Kirkegaard H, Johnsen NF, Christensen J, et al. Association of adherence to lifestyle recommendations and risk of colorectal cancer: a prospective Danish cohort study. BMJ. 2010;341:c5504.

36. Platz EA, Willett WC, Colditz GA, et al. Proportion of colon cancer risk that might be preventable in a cohort of middle-aged US men. Cancer Causes Control. 2000;11(7):579-588.

37. Aleksandrova K, Pischon T, Jenab M, et al. Combined impact of healthy lifestyle factors on colorectal cancer: a large European cohort study. BMC Med. 2014;12(1):168.

38. Hang J, Cai B, Xue P, et al. The joint effects of lifestyle factors and comorbidities on the risk of colorectal cancer: a large Chinese retrospective case-control study. PLoS One. 2015;10(12):e0143696.

39. Tabung FK, Steck SE, Burch JB, et al. A healthy lifestyle index is associated with reduced risk of colorectal adenomatous polyps among non-users of non-steroidal antiinflammatory drugs. J Prim Prev. 2015;36(1):21-31.

40. Buckland G, Travier N, Huerta JM, et al. Healthy lifestyle index and risk of gastric adenocarcinoma in the EPIC cohort study. Int J Cancer. 2015;137(3):598-606.

41. McKenzie F, Ferrari P, Freisling H, et al. Healthy lifestyle and risk of breast cancer among postmenopausal women in the European Prospective Investigation into Cancer and Nutrition cohort study. Int J Cancer. 2015;136(11):26402648.

42. McKenzie F, Ellison-Loschmann L, Jeffreys M, et al. Healthy lifestyle and risk of breast cancer for indigenous and non-indigenous women in New Zealand: a case control study. BMC Cancer. 2014;14(1):12.

43. Sánchez-Zamorano LM, Flores-Luna L, Ángeles-Llerenas A, et al. Healthy lifestyle on the risk of breast cancer. Cancer Epidemiol Biomarkers Prev. 2011;20(5):912-922.

44. Wakasugi M, Kazama JJ, Narita I, et al. Association between combined lifestyle factors and non-restorative sleep in Japan: a cross-sectional study based on a Japanese health database. PLoS One. 2014;9(9):e108718.

45. Hartman L, Van Dongen J, Hildebrandt V, Strijk J. The role of vitality in the relationship between a healthy lifestyle and societal costs of health care and lost productivity: a mediation analysis. Am J Health Promot. 2016;30(6):465-474.

46. Blake H, Stanulewicz N, Griffiths K. Healthy lifestyle behaviors and health promotion attitudes in preregistered nurses: a questionnaire study. J Nurs Educ. 2017;56(2):94103.

47. Kozłowska K, Szczecińska A, Roszkowski W, et al. Patterns of healthy lifestyle and positive health attitudes in older Europeans. J Nutr Health Aging. 2008;12(10):728733.

48. Kaleta D, Makowiec-Dąbrowska T, Jegier A. Lifestyle index and work ability. Int J Occup Med Environ Health. 2006;19(3):170-177.

49. Lucini D, Zanuso S, Blair S, Pagani M. A simple healthy lifestyle index as a proxy of wellness: a proof of concept. Acta Diabetol. 2015;52(1):81-89.

50. Schröder H, Ramos R, Baena-Díez JM, et al. Determinants of the transition from a cardiometabolic normal to abnormal overweight/obese phenotype in a Spanish population. Eur J Nutr. 2014;53(6):1345-1353.

51. Sowa A, Tobiasz-Adamczyk B, Topór-Mądry R, et al. Predictors of healthy ageing: public health policy targets. BMC Health Serv Res. 2016;16(5):289.

52. Gathirua-Mwangi W, Cohee A, Tarver WL, et al. Factors associated with adherence to mammography screening among insured women differ by income levels. Womens Health Issues. 2018;28(5):462-469.

53. Champion VL, Rawl SM, Bourff SA, et al. Randomized trial of DVD, telephone, and usual care for increasing mammography adherence. J Health Psychol. 2016;21(6):916-926.

54. Gathirua-Mwangi WG, Monahan PO, Stump T, et al. Mammography adherence in African-American women: results of a randomized controlled trial. Ann Behav Med. 2015;50(1):70-78.

55. Champion VL, Christy SM, Rakowski W, et al. A randomized trial to compare a tailored web-based intervention and tailored phone counseling to usual care for increasing colorectal cancer screening. Cancer Epidemiol Biomarkers Prev. 2018;27(12):1433-1441.

56. US Department of Health and Human Services and US Department of Agriculture. 2015-2020 Dietary Guidelines for Americans. $8^{\text {th }}$ ed. Available at: https://health.gov/ dietaryguidelines/2015/guidelines/. Accessed August 22, 2018.

57. US Centers for Disease Control and Prevention. How much physical activity do adults need? Available at: https://www.cdc.gov/physicalactivity/basics/adults/index.htm. Accessed June 15, 2018.

58. O’Keefe JH, Bybee KA, Lavie CJ. Alcohol and cardiovas- 
cular health: the razor-sharp double-edged sword. $J A m$ Coll Cardiol. 2007;50(11):1009-1014.

59. Klatsky AL, Udaltsova N. Alcohol drinking and total mortality risk. Ann Epidemiol. 2007;17(5):S63-S67.

60. Fedirko V, Tramacere I, Bagnardi V, et al. Alcohol drinking and colorectal cancer risk: an overall and dose-response meta-analysis of published studies. Ann Oncol. 2011;22(9):1958-1972.

61. Bagnardi V, Blangiardo M, La Vecchia C, Corrao G. A meta-analysis of alcohol drinking and cancer risk. $\mathrm{Br} \mathrm{J}$ Cancer. 2001;85(11):1700-1705.

62. Corrao G, Bagnardi V, Zambon A, La Vecchia C. A metaanalysis of alcohol consumption and the risk of 15 diseases. Prev Med. 2004;38(5):613-619.

63. Longnecker MP. Alcoholic beverage consumption in relation to risk of breast cancer: meta-analysis and review. Cancer Causes Control. 1994;5(1):73-82.

64. Willett WC, Stampfer MJ, Colditz GA, et al. Moderate alcohol consumption and the risk of breast cancer. $N$ Engl JMed. 1987;316(19):1174-1180.

65. Allen NE, Beral V, Casabonne D, et al. Moderate alcohol intake and cancer incidence in women. J Natl Cancer Inst. 2009;101(5):296-305.

66. Chen WY, Rosner B, Hankinson SE, et al. Moderate alcohol consumption during adult life, drinking patterns, and breast cancer risk. JAMA. 2011;306(17):1884-1890.

67. Griswold MG, Fullman N, Hawley C, et al. Alcohol use and burden for 195 countries and territories, 1990-2016: a systematic analysis for the Global Burden of Disease Study 2016. Lancet. 2018;392(10152):1015-1035.

68. US Centers for Disease Control and Prevention. Defining Adult Overweight and Obesity. Available at: https:// www.cdc.gov/obesity/adult/defining.html. Accessed June 10, 2018.

69. Lee K, Lim HT, Park SM. Factors associated with use of breast cancer screening services by women aged $\geq 40$ years in Korea: the Third Korea National Health and Nutrition Examination Survey 2005 (KNHANES III). BMC Cancer. 2010;10(1):144.

70. Sözmen K, Unal B, Sakarya S, et al. Determinants of breast and cervical cancer screening uptake among women in turkey. Asia Pac J Public Health. 2016;28(6):528538.

71. Rosenberg SM, Moskowitz CS, Ford JS, et al. Health care utilization, lifestyle, and emotional factors and mammography practices in the Childhood Cancer Survivor Study. Cancer Epidemiol Biomarkers Prev. 2015;24(11):16991706.

72. Komenaka IK, Nodora JN, Hsu C-H, et al. Association of health literacy with adherence to screening mammography guidelines. Obstet Gynecol. 2015;125(4):852-859.

73. Beeken RJ, Wilson R, McDonald L, Wardle J. Body mass index and cancer screening: findings from the English Longitudinal Study of Ageing. J Med Screen. 2014;21(2):76-81.

74. Byles J, Leigh L, Chojenta C, Loxton D. Adherence to recommended health checks by women in mid-life: data from a prospective study of women across Australia. Aust NZ J Public Health. 2014;38(1):39-43.

75. Shippee ND, Mullan RJ, Nabhan M, et al. Adherence to preventive recommendations: experience of a cohort presenting for executive health care. Popul Health Manag.
2012;15(2):65-70.

76. Caleffi M, Ribeiro RA, Júnior AJB, et al. Adherence to a breast cancer screening program and its predictors in underserved women in southern Brazil. Cancer Epidemiol Biomarkers Prev. 2010;19(10):2673-2679.

77. Katz D, Tengekyon AJ, Kahan NR, Calderon-Margalit R. Patient and physician characteristics affect adherence to screening mammography: a population-based cohort study. PLoS One. 2018;13(3):e0194409.

78. Tworek C, Nadpara P, Adkins B, et al. Smoking and breast cancer screening in West Virginia: opportunities for intervention. WV Med J. 2009;105 Spec No:48-53.

79. Brown KC, Fitzhugh EC, Neutens JJ, Klein DA. Screening mammography utilization in Tennessee women: the association with residence. J Rural Health. 2009;25(2):167173.

80. Dimitrakaki C, Boulamatsis D, Mariolis A, et al. Use of cancer screening services in Greece and associated social factors: results from the nation-wide Hellas Health I survey. Eur J Cancer Prev. 2009;18(3):248-257.

81. Eichholzer M, Richard A, Rohrmann S, et al. Overweight, obesity, and breast cancer screening: results from the 2012 Swiss Health Survey. Eur J Cancer Prev. 2016;25(2):130-136.

82. Beckmann KR, Roder DM, Hiller JE, et al. Do breast cancer risk factors differ among those who do and do not undertake mammography screening? J Med Screen. 2013;20(4):208-219.

83. Hirth JM, Laz TH, Rahman M, Berenson AB. Racial/ethnic differences affecting adherence to cancer screening guidelines among women. J Womens Health. 2016;25(4):371-380.

84. Miller S, Rafanan L, Keihany S, Reyes-Ortiz CA. The relationship between body mass index and cancer screening utilization among older women in Latin American and Caribbean cities. J Women Aging. 2015;27(1):3-16.

85. Bussière C, Sicsic J, Pelletier-Fleury N. The effects of obesity and mobility disability in access to breast and cervical cancer screening in france: results from the national health and disability survey. PLoS One. 2014;9(8):e104901.

86. Tekkel M, Veideman T, Rahu M. Use of mammography, pap test and prostate examination by body mass index during the developmental period of cancer screening in Estonia. Public Health. 2011;125(10):697-703.

87. Banerjea R, Findley PA, Sambamoorthi U. Disparities in preventive care by body mass index categories among women. Women Health. 2008;47(4):1-17.

88. Hellmann SS, Njor SH, Lynge E, et al. Body mass index and participation in organized mammographic screening: a prospective cohort study. BMC Cancer. 2015;15:294.

89. Hernandez-Boussard T, Ahmed SM, Morton JM. Obesity disparities in preventive care: findings from the National Ambulatory Medical Care Survey, 2005-2007. Obesity (Silver Spring). 2012;20(8):1639-1644.

90. Kim H, Filson C, Joski P, et al. Association between online information-seeking and adherence to guidelines for breast and prostate cancer screening. Prev Chronic Dis. 2018; 15:E45.

91. Bowser D, Marqusee H, El Koussa M, Atun R. Health system barriers and enablers to early access to breast cancer screening, detection, and diagnosis: a global analysis applied to the MENA region. Public Health. 2017;152:58- 
74.

92. Freund A, Cohen M, Azaiza F. Factors associated with routine screening for the early detection of breast cancer in cultural-ethnic and faith-based communities. Ethn Health. 2017:1-17.

93. Morman NA, Byrne L, Collins C, et al. Breast cancer risk assessment at the time of screening mammography: perceptions and clinical management outcomes for women at high risk. J Genet Couns. 2017;26(4):776-784.

94. Peterson EB, Ostroff JS, DuHamel KN, et al. Impact of provider-patient communication on cancer screening adherence: a systematic review. Prev Med. 2016;93:96-105.

95. Maruthur NM, Bolen S, Brancati FL, Clark JM. Obesity and mammography: a systematic review and meta-analysis. J Gen Intern Med. 2009;24(5):665-677.

96. Aldrich T, Hackley B. The impact of obesity on gynecologic cancer screening: an integrative literature review. $J$ Midwifery Womens Health. 2010;55(4):344-356.

97. Oeffinger KC, Fontham E, Etzioni R, et al. Breast cancer screening for women at average risk: 2015 guideline update from the American Cancer Society. JAMA. 2015;314(15):1599-1614.

98. US Centers for Disease Control and Prevention. Fact Sheets - Moderate Drinking. Available at: https://www. cdc.gov/alcohol/fact-sheets/moderate-drinking.htm. Accessed August 22, 2018. 